\title{
3 Research Square

\section{Elevated Mid-trimester 4-hour Postprandial Triglycerides for Predicting Late-onset Preeclampsia and Gestational Hypertension: a Prospective Screening Study}

Qin Liu

Tongji University Shanghai First Maternal and Infant Hospital

Zhihong Zhu

Zhongshan Hospital Fudan University

Wen Cai

Shanghai General Hospital

Liu Yang

Zhongshan Hospital Fudan University

Shuangdi Li

Tongji University Shanghai First Maternal and Infant Hospital

Jiarong Zhang ( $\square$ maggie1974@126.com )

Zhongshan Hospital Fudan University https://orcid.org/0000-0002-6627-9169

\section{Research Article}

Keywords: hypertriglyceridemia, preeclampsia, gestational hypertension, OLTT, prediction, metabolism

Posted Date: November 3rd, 2021

DOl: https://doi.org/10.21203/rs.3.rs-904603/v1

License: (c) (i) This work is licensed under a Creative Commons Attribution 4.0 International License.

Read Full License 


\section{Abstract}

Background: Abnormal maternal lipid concentrations are associated with increased risk of preeclampsia. However, previous studies mainly focused on fasting lipid concentrations, scarce data have been published on the relationship between postprandial triglyceride (TG) concentrations in the second trimester and the risk of preeclampsia. Our aim is to evaluate the potential of triglyceride (TG) concentrations at the time of oral lipid tolerance test (OLTT) measurement in the second trimester to predict preeclampsia and to elucidate the lipid metabolic changes related to these diseases.

Methods: This is a prospective cohort study of Pregnant women at 12-24 weeks of gestation undergone an OLTT in a university affiliated hospital between May 2019 and January 2020. Data were stratified into binaries according to the OLTT results. The receiver operating characteristic (ROC) curve analysis was conducted to determine the optimal cut-off points of TG, HDL-C, LDL-C, sd-LDL, FFA, and BG for predicting preeclampsia.

Results: 438 pregnant women were recruited to undergo an OLTT at 12-24 weeks of gestation. Among these, 24 women developed preeclampsia and 414 women remained normotensive. Women who subsequently developed preeclampsia had higher concentrations of 4-h postprandial TG than those who remained normotensive. In the linear logistic regression analyses of potential confounding factors, midtrimester 4-h postprandial TG concentrations at the time of OLTT measurement were significantly higher in preeclamptic cases than in controls.

Conclusions: Dyslipidemia in the second trimester of pregnancy, particularly postprandial hypertriglyceridemia, appears to be associated with an increased risk of preeclampsia. Mid-trimester 4-h postprandial TG concentration at the time of OLTT measurement may be a potential predictive marker of preeclampsia.

\section{Background}

Preeclampsia is a multifaceted condition, and its underlying etiologies are still poorly defined. Preeclampsia is associated with high morbidity and mortality; it affects $5 \%-7 \%$ of all pregnant women and is responsible for $>70,000$ maternal and 500,000 fetal deaths annually worldwide (1). It is a leading cause of maternal death, severe maternal morbidity, maternal intensive care admissions, cesarean section, and premature birth. Hypertensive disorders of pregnancy, in particular preeclampsia, are also recognized as a major risk factor for cardiovascular disease later in life for both the woman and her child (2).

The increased risks of preeclampsia are associated with maternal obesity, polycystic ovarian syndrome, and pre-existing and gestational diabetes mellitus (GDM). These are all syndromes of maternal metabolic disturbance and, to varying degrees, abnormal maternal lipid concentrations (3). According to a recent systematic review, including 29 studies, hypertriglyceridemia preceded the onset of preeclampsia. More specifically, within the meta-analysis of five cohort studies, hypertriglyceridemia during the second 
trimester was associated with the development of preeclampsia, overall suggesting that hypertriglyceridemia may be involved in the development of preeclampsia (4). Increasingly, the role of lipids has been recognized as critically important in vascular risk modification, and it is possible, therefore, that lipids in pregnancy has an impact on placental vascular development, which may be critical in the development of preeclampsia (5).

During pregnancy, the lipid concentrations increase gradually, resulting in a physiological hyperlipidemia state. Previous studies mainly focused on fasting lipid concentrations, neglected that we're in a postprandial state in most of the day $(6,7)$. Postprandial dyslipidemia can reflect the decreased ability of pregnant women to metabolize and clear lipids, which occurs before fasting dyslipidemia. However, only a few studies have characterized the relationship between postprandial triglyceride (TG) concentrations in the second trimester and the risks of preeclampsia.

The postprandial response to an oral lipid tolerance test (OLTT) is a better metabolic challenge to emulate nutrient overload in comparison to an oral glucose tolerance test (OGTT). OLTT is used for screening dyslipidemia (8). Here, we tested the hypothesis that elevated postprandial TG concentrations are correlated with an increased likelihood of preeclampsia in Chinese women. We aimed to determine whether pregnant women with abnormal OLTT results during the second trimester are at a high risk of developing preeclampsia.

\section{Materials And Methods}

\section{Participants and ethical statement}

This prospective cohort study enrolled 506 pregnant women who attended the prenatal examination clinics in Shanghai General Hospital between May 2019 and January 2020. In the present analysis, we excluded one woman who had a miscarriage in the second trimester, and 67 women who met the following exclusion criteria: (1) multiple pregnancy; (2) unnatural pregnancy; (3) polycystic ovarian syndrome, thyroid disease, diabetes, heart disease, or hyperlipidemia; (4) combined with autoimmune diseases; (5) combined with intrahepatic cholestasis of pregnancy or viral hepatitis; and (6) fasting TG concentrations > 5.6 (mmol/L). The remaining 439 women were included in the final analysis (Fig. 1). These subjects were recruited to undergo an OLTT at 12-24 gestational weeks. A unified standard fat meal referring to the guide was provided for the subjects; the calorie of which was approximately 680 $\mathrm{kcal}$, consisting of a 50-g egg, 30-g butter, $100-\mathrm{g}$ two pieces of toast bread and $250-\mathrm{ml}$ whole milk. The energy proportion of fat, protein, and carbohydrate were $59 \%, 12 \%$ and $29 \%$, respectively (8). TG, free fat acid (FFA), small dense low-density lipoprotein (sd-LDL) were measured by blood sampling at fasting, and TG was measured at $4 \mathrm{~h}$ after the fat meal. Each enrolled pregnant woman underwent a 75-g OGTT at 24-24 weeks of gestation. In addition, fasting lipid concentrations, including serum total cholesterol (TC), TGs, high-density lipoprotein-cholesterol (HDL-C), and low-density lipoprotein-cholesterol (LDL-C) as well as insulin, alanine aminotransferase (ALT), and aspartate aminotransferase (AST) concentrations were measured. Women were diagnosed with GDM if one or more of the following plasma glucose values 
during the 75-g OGTT at 24-28 gestational weeks were met or exceeded: $0 \mathrm{~h}, 5.1 \mathrm{mmol} / \mathrm{L} ; 1 \mathrm{~h}, 10.0$ $\mathrm{mmol} / \mathrm{L}$; and $2 \mathrm{~h}, 8.5 \mathrm{mmol} / \mathrm{L}$. Each patient provided informed consent, and the research was conducted in compliance with the Declaration of Helsinki. This trial has been registered in Chinese Clinical Trial Registry (chiCTR1800018884). The study protocol was approved by the ethics committee of Shanghai General Hospital, Shanghai Jiao Tong University (approval No.: JRS [2018] No.26; approval date: June 20, 2018).

\section{Grouping}

All pregnant women were categorized into the following binaries according to the OLTT results at the midtrimester: low binary [fasting TG (FTG) $<2.4 \mathrm{mmol} / \mathrm{L}$ (median); $4 \mathrm{~h}, \mathrm{TG}<3.47 \mathrm{mmol} / \mathrm{L}$ (median)] and high binary ( $F T G \geq 2.4 \mathrm{mmol} / \mathrm{L} ; 4 \mathrm{~h}, \mathrm{TG} \geq 3.47 \mathrm{mmol} / \mathrm{L}$ ) groups. The women were also categorized into the following two groups according to the blood pressure levels and the presence or absence of proteinuria: normal and preeclampsia groups (Fig. 1).

\section{Data collection}

The following maternal characteristics were assessed: age at delivery, height, body weight before pregnancy, blood pressure, and insulin treatment during pregnancy.

\section{Metabolic measurements}

Plasma glucose (PG) concentrations were measured enzymatically. Fasting insulin (FINS) and serum lipid (TC, TG, HDL-C, LDL-C, sd-LDL, and FFA) concentrations were determined by chemiluminescent assays. Hemoglobin $\mathrm{A} 1 \mathrm{C}$ was determined by high-performance liquid chromatography. Homeostasis model assessment for $\beta$ cell function (HOMA- $\beta$ ) and homeostasis model assessment for insulin resistance index (HOMA-IR) were calculated to evaluate $\beta$ cell function and insulin resistance using the following formulas: $\mathrm{HOMA}-\beta=20 \times \mathrm{FINS}(\mu \mathrm{U} / \mathrm{mL}) / \mathrm{FPG}(\mathrm{mmol} / \mathrm{L})-3.5)$ and $\mathrm{HOMA}-\mathrm{IR}=\mathrm{FPG}(\mathrm{mmol} / \mathrm{L}) \times$ FINS $(\mu \mathrm{U} / \mathrm{mL}) / 22.5$.

\section{Statistical analysis}

Data were expressed as the mean \pm standard deviation for normally distributed variables and as the median with the interquartile range for skewed data. To determine the differences between the groups, we conducted an analysis of variance or the Kruskal-Wallis test for continuous variables and a chi-square test for categorical variables. Least significant difference tests were used to perform pairwise comparisons between two groups. The receiver operating characteristic $(\mathrm{ROC})$ curve analysis was conducted to determine the optimal cut-off points of TG, HDL-C, LDL-C, sd-LDL, FFA, and BG for predicting preeclampsia. Each optimal cut-off point was assessed by searching for the maximum value of sensitivity + specificity-1 (Youden index). The area under curve (AUC) was calculated to evaluate the predictive powers. Logistic regression analysis was performed to determine whether the elevated postprandial TG concentrations during mid-gestation were independently associated with preeclampsia. Statistical significance was set at $p<0.05$. Statistical analyses were performed using SPSS (IBM Corp., Armonk, NY). 


\section{Results}

Demographic and metabolic characteristics of all patients in the second trimester who were categorized into binaries according to TG concentrations at the time of OLTT measurement

There were significant differences in the metabolic levels during pregnancy between the high and low fasting TG groups. The high fasting TG binary group had a higher pre-pregnancy body mass index (BMI) $\left(22.39 \pm 2.11\right.$ versus $\left.20.82 \pm 1.78 \mathrm{~kg} / \mathrm{m}^{2}, p<0.01\right)$, higher fasting PG concentrations $(4.71 \pm 0.46$ versus $4.59 \pm 0.35 \mathrm{mmol} / \mathrm{L}, p=0.03)$, higher HOMA-IR (1.60 (1.14-2.23) versus 1.23 (1.01-1.66), $p<0.01)$, greater neonatal weight $(3318.93 \pm 418.02$ versus $3260.50 \pm 415.58 \mathrm{~g}, p=0.03)$, and lower HDL-C concentrations $(1.84 \pm 0.35$ versus $2.05 \pm 0.39 \mathrm{mmol} / \mathrm{L}, p<0.01)$ than the low fasting TG binary group (Table 1). 
Table 1

Demographic and metabolic characteristics of all pregnant women at 12-24 gestational weeks who were categorized into binaries according to their fasting TG concentrations at the time of OLTT measurement

\begin{tabular}{|c|c|c|c|}
\hline \multirow[t]{3}{*}{ Variables } & Low binary & High binary & \multirow[t]{3}{*}{$p$ value } \\
\hline & $(\mathrm{FTG}<2.4 \mathrm{mmol} / \mathrm{L})$ & $(\mathrm{FTG} \geq 2.4 \mathrm{mmol} / \mathrm{L})$ & \\
\hline & $(N=219)$ & $(N=219)$ & \\
\hline Age (years) & $30.95 \pm 3.7$ & $30.68 \pm 4.03$ & 0.61 \\
\hline Gravidity & $2.32 \pm 1.12$ & $2.31 \pm 1.29$ & 0.95 \\
\hline Parity & $0.54 \pm 0.34$ & $0.56 \pm 0.72$ & 0.91 \\
\hline Prepregnancy BMI $\left(\mathrm{kg} / \mathrm{m}^{2}\right)$ & $20.82(19.59-23.15)$ & $22.39(20.62-24.83)$ & $<0.01$ \\
\hline Systolic Blood Pressure (mmHg) & $113.29 \pm 14.51$ & $113.9 \pm 10.36$ & 0.71 \\
\hline Diastolic Blood Pressure (mmHg) & $68.04 \pm 8.23$ & $67.48 \pm 10.34$ & 0.66 \\
\hline HbA1c (\%) & $5.00(4.90-5.20)$ & $5.10(4.90-5.30)$ & 0.29 \\
\hline $\mathrm{FPG}(\mathrm{mmol} / \mathrm{L})$ & $4.59 \pm 0.35$ & $4.71 \pm 0.46$ & 0.03 \\
\hline 1h PG (mmol/L) & $7.94 \pm 1.75$ & $8.28 \pm 1.76$ & 0.16 \\
\hline 2h PG (mmol/L) & $7.00 \pm 1.41$ & $7.11 \pm 1.31$ & 0.52 \\
\hline Total Cholesterol (mmol/L) & $5.88(5.45-6.55)$ & $5.90(5.41-6.78)$ & 0.71 \\
\hline $\mathrm{HDL}-\mathrm{C}(\mathrm{mmol} / \mathrm{L})$ & $2.05 \pm 0.39$ & $1.84 \pm 0.35$ & $<0.01$ \\
\hline LDL-C (mmol/L) & $3.14 \pm 0.79$ & $3.04 \pm 0.97$ & 0.42 \\
\hline HOMA- $\beta$ (\%) & $124.21(86.01-159.49)$ & 122.88 (94.77-171.74) & 0.12 \\
\hline HOMA-IR & $1.23(1.01-1.66)$ & $1.60(1.14-2.23)$ & $<0.01$ \\
\hline Neonatal weight (g) & $3260.50 \pm 415.58$ & $3318.93 \pm 418.02$ & 0.03 \\
\hline preeclampsia & $4(0.037)$ & $8(0.073)$ & 0.229 \\
\hline Adverse Neonatal Outcomes & $26(0.239)$ & $30(0.273)$ & 0.56 \\
\hline $\begin{array}{l}\text { Data are expressed as the mean } \pm \\
\text { body mass index; FPG: fasting pla } \\
\text { lipoprotein-cholesterol; HOMA-IR: } h \\
\beta \text { : homeostasis model assessmen } \\
\text { triglyceride; } 1 \mathrm{hPG} \text { : } 1 \text {-h plasma gluc }\end{array}$ & $\begin{array}{l}\text { dard deviation or medi } \\
\text { glucose; HbA1c: hemo } \\
\text { ostasis model assessn } \\
\text { B cell function; LDL-C: I } \\
\text { 2hPG: 2-h plasma gluc }\end{array}$ & $\begin{array}{l}\text { terquartile range), or } \mathrm{n} \\
\text { in } \mathrm{A} 1 \mathrm{c} ; \mathrm{HDL}-\mathrm{C} \text { : high-den } \\
\text { for insulin resistance in } \\
\text { density lipoprotein-chole }\end{array}$ & $\begin{array}{l}\text {.BMI: } \\
\text { y; HOMA } \\
\text { erol; TG: }\end{array}$ \\
\hline
\end{tabular}

Although there was no difference in pre-pregnancy BMI, there were significant differences in the metabolic levels during pregnancy in between the high and low 4-h postprandial TG groups. The high 4-h TG binary group had higher HOMA-IR (1.58 (1.15-2.23) versus $1.23(0.96-1.71), p<0.01)$, higher fasting 
PG concentrations $(4.75 \pm 0.47$ versus $4.60 \pm 0.34 \mathrm{mmol} / \mathrm{L}, p=0.01)$, and lower HDL-C concentrations ( $1.83 \pm 0.38$ versus $2.07 \pm 0.39 \mathrm{mmol} / \mathrm{L}, p<0.01)$, and lower HDL-C concentrations $(2.95 \pm 1.00$ versus $3.14 \pm 0.76 \mathrm{mmol} / \mathrm{L}, p=0.04$ ) than the low $4-\mathrm{h}$ TG binary group (Table 2). In addition, the prevalence rates of preeclampsia were $1.83 \%$ and $9.09 \%(p=0.015)$ in the two groups $(4-\mathrm{h} \mathrm{TG}<3.47 \mathrm{mmol} / \mathrm{L}$ and $4-\mathrm{h} \mathrm{TG}$ $\geq 3.47 \mathrm{mmol} / \mathrm{L}$, respectively) (Table 2 ). 
Table 2

Demographic and metabolic characteristics of all pregnant women at 12-24 gestational weeks who were categorized into binaries according to their 4-h postprandial TG concentrations at the time of OLTT measurement

\begin{tabular}{|c|c|c|c|}
\hline \multirow[t]{3}{*}{ Variables } & Low binary & High binary & \multirow[t]{3}{*}{$p$ value } \\
\hline & \multicolumn{2}{|l|}{ ( $4 \mathrm{~h} \mathrm{TG}<3.47 \mathrm{mmol} / \mathrm{L}$ ) } & \\
\hline & $(N=219)$ & $(N=219)$ & \\
\hline Age (years) & $30.98 \pm 3.80$ & $30.58 \pm 3.99$ & 0.77 \\
\hline Gravidity & $2.39 \pm 1.19$ & $2.23 \pm 1.22$ & 0.33 \\
\hline Parity & $0.56 \pm 0.80$ & $0.53 \pm 0.71$ & 0.49 \\
\hline Prepregnancy BMI $\left(\mathrm{kg} / \mathrm{m}^{2}\right)$ & $21.45(19.81-23.53)$ & $21.78(20.31-24.45)$ & 0.34 \\
\hline Systolic Blood Pressure (mmHg) & $114.93 \pm 11.26$ & $113.49 \pm 11.05$ & 0.84 \\
\hline Diastolic Blood Pressure (mmHg) & $68.17 \pm 7.73$ & $68.08 \pm 10.60$ & 0.76 \\
\hline $\mathrm{HbA1c}(\%)$ & $5.0(4.9-5.3)$ & $5.1(4.9-5.3)$ & 0.1 \\
\hline $\mathrm{FPG}(\mathrm{mmol} / \mathrm{L})$ & $4.60 \pm 0.34$ & $4.75 \pm 0.47$ & 0.01 \\
\hline 1h PG (mmol/L) & $7.87 \pm 1.71$ & $8.22 \pm 1.87$ & 0.16 \\
\hline 2h PG (mmol/L) & $6.70 \pm 1.31$ & $7.03 \pm 1.43$ & 0.35 \\
\hline Total Cholesterol (mmol/L) & $5.98(5.61-6.68)$ & $5.76(5.29-6.66)$ & 0.14 \\
\hline $\mathrm{HDL}-\mathrm{C}(\mathrm{mmol} / \mathrm{L})$ & $2.07 \pm 0.39$ & $1.83 \pm 0.38$ & $<0.01$ \\
\hline LDL-C (mmol/L) & $3.14 \pm 0.76$ & $2.95 \pm 1.00$ & 0.04 \\
\hline HOMA- $\beta(\%)$ & $124.53(85.85-164.40)$ & $121.32(96.58-168.19)$ & 0.29 \\
\hline HOMA-IR & $1.23(0.96-1.71)$ & $1.58(1.15-2.23)$ & $<0.01$ \\
\hline Neonatal weight (g) & $3270.34 \pm 428.62$ & $3308.29 \pm 407.14$ & 0.08 \\
\hline preeclampsia & $4(0.018)$ & $20(0.091)$ & 0.015 \\
\hline Adverse Neonatal Outcomes & $48(0.220)$ & $64(0.291)$ & 0.23 \\
\hline $\begin{array}{l}\text { Data are expressed as the mean } \pm \\
\text { body mass index; FPG: fasting pla } \\
\text { lipoprotein-cholesterol; HOMA-IR: } h \\
\beta \text { : homeostasis model assessmen } \\
\text { triglyceride; } 1 \mathrm{hPG} \text { : } 1 \text {-h plasma gluc }\end{array}$ & $\begin{array}{l}\text { hdard deviation or medi } \\
\text { a glucose; HbA1c: hemo } \\
\text { eostasis model assessi } \\
\text { } \beta \text { cell function; LDL-C: } \\
; 2 \text { hPG: } 2 \text {-h plasma gluc }\end{array}$ & $\begin{array}{l}\text { (interquartile range), or n (\% } \\
\text { bin A1c; } \mathrm{HDL}-\mathrm{C} \text { : high-densit } \\
\text { th for insulin resistance inde } \\
\text {--density lipoprotein-cholest } \\
\text { e }\end{array}$ & $\begin{array}{l}\text { BMl: } \\
\text {; HOMA- } \\
\text { ol; TG: }\end{array}$ \\
\hline
\end{tabular}

Demographic and metabolic characteristics of preeclampsia in the second trimester 
Maternal plasma lipid and lipoprotein concentrations are shown in Table 3. Women who subsequently developed preeclampsia had higher plasma glucose and 4-h postprandial TG concentrations and HOMAIR than the normotensive group. 
Table 3

Demographic and metabolic characteristics of preeclamptic cases and normotensive controls at 12-24 gestational weeks

\begin{tabular}{|c|c|c|c|}
\hline \multirow[t]{3}{*}{ Variables } & Preeclampsia & Control & \multirow[t]{3}{*}{$p$ value } \\
\hline & $(N=24)$ & $(N=414)$ & \\
\hline & $($ Mean \pm SEM $)$ & (Mean \pm SEM ) & \\
\hline Age (years) & $30.76 \pm 3.89$ & $31.83 \pm 4.19$ & 0.35 \\
\hline Gravidity & $2.31 \pm 1.21$ & $2.23 \pm 1.01$ & 0.90 \\
\hline Parity & $0.53 \pm 0.21$ & $0.54 \pm 0.15$ & 0.81 \\
\hline Prepregnancy BMI (kg/m2) & $8.07 \pm 9.71$ & $8.49 \pm 12.06$ & 0.89 \\
\hline Systolic Blood Pressure (mmHg) & $112.81 \pm 12.24$ & $128.12 \pm 10.40$ & 0.01 \\
\hline Diastolic Blood Pressure (mmHg) & $67.27 \pm 9.18$ & $76.72 \pm 7.14$ & 0.01 \\
\hline HbA1c (\%) & $5.06 \pm 0.36$ & $5.24 \pm 0.23$ & 0.09 \\
\hline $\mathrm{FPG}(\mathrm{mmol} / \mathrm{L})$ & $5.00(4.70 \pm 5.22)$ & $4.65(4.37 \pm 4.86)$ & 0.001 \\
\hline 1h PG (mmol/L) & $9.62(8.34 \pm 10.59)$ & $7.94(6.73 \pm 9.12)$ & 0.002 \\
\hline 2h PG (mmol/L) & $7.06(6.47 \pm 7.95)$ & $6.91(6.10 \pm 7.81)$ & 0.51 \\
\hline Total Cholesterol (mmol/L) & $5.5(5.28 \pm 6.06)$ & $5.96(5.48 \pm 6.74)$ & 0.245 \\
\hline $\mathrm{HDL}-\mathrm{C}(\mathrm{mmol} / \mathrm{L})$ & $1.79(1.41 \pm 2.18)$ & $1.92(1.66 \pm 2.21)$ & 0.364 \\
\hline LDL-C (mmol/L) & $2.42(1.96 \pm 3.24)$ & $3.13(2.50 \pm 3.57)$ & 0.044 \\
\hline FFA & $0.36(0.28 \pm 0.89)$ & $0.41(0.28 \pm 0.80)$ & 0.95 \\
\hline sd-LDL & $40.96(32.80 \pm 55.52)$ & $41.13(31.71 \pm 53.65)$ & 0.72 \\
\hline HOMA- $\beta$ (\%) & $121.34(104.61 \pm 133.48)$ & $123.06(92.38 \pm 165.20)$ & 0.606 \\
\hline HOMA-IR & $2.25(1.52 \pm 2.86)$ & $1.34(1.04 \pm 1.83)$ & 0.004 \\
\hline FTG & $2.81(2.28 \pm 3.30)$ & $2.35(1.88 \pm 3.04)$ & 0.12 \\
\hline $4 \mathrm{~h} \mathrm{TG}$ & $4.20(3.63 \pm 5.12)$ & $3.38(2.44 \pm 4.33)$ & 0.037 \\
\hline Neonatal weight $(\mathrm{g})$ & $3312.63 \pm 405.22$ & $2961.66 \pm 541.78$ & 0.05 \\
\hline $\begin{array}{l}\text { Data are expressed as the mean } \pm \\
\text { plasma glucose; FTG: fasting trigl } \\
\text { cholesterol; HOMA-IR: homeostasi } \\
\text { homeostasis model assessment fo } \\
\text { fasting small dense low density lip } \\
\text { glucose; } 2 \mathrm{~h} \text { PG: } 2 \text { h plasma glucos }\end{array}$ & $\begin{array}{l}\text { EM. BMI: body mass index; } \\
\text { eride; HbA1c: hemoglobin A } \\
\text { nodel assessment for insul } \\
\beta \text { cell function; LDL-C: low-c } \\
\text { protein; SEM: standard error } \\
\text { 4h TG: 4-h triglyceride }\end{array}$ & $\begin{array}{l}\text { A: fasting free fat acid; } \\
\text {; HDL-C: high-density lipo } \\
\text { resistance index; HOMA- } \\
\text { nsity lipoprotein-cholester } \\
\text { f the mean; } 1 \mathrm{~h} \mathrm{PG:} 1 \mathrm{~h} \text { pla }\end{array}$ & $\begin{array}{l}\text { : fasting } \\
\text { otein- } \\
\text { sd-LDL: } \\
\text { na }\end{array}$ \\
\hline
\end{tabular}


Table 4 presents the optimal cut-off points of maternal lipid concentrations in the second trimester for predicting preeclampsia. Four-hour postprandial TG concentrations predicting preeclampsia had the strongest predictive power with the largest AUC [0.680 (95\% confidence interval [Cl]: 0.505-0.854)]. The optimal cut-off points for second-trimester FTG and 4-h postprandial TG in predicting preeclampsia were $\geq 2.93$ and $3.92 \mathrm{mmol} / \mathrm{L}$, respectively. Besides, the optimal cut-off points for LDL-C, FFA, sd-LDL, and $\mathrm{HDL}-\mathrm{C}$ in predicting preeclampsia were $\geq 1.8,1.46,29.085$ and $\leq 2.42 \mathrm{mmol} / \mathrm{L}$, respectively. Moreover, only high 4-h postprandial TG in the second trimester was significantly associated with the morbidity of preeclampsia $(p=0.045)$.

Table 4

Optimal cut-off points of maternal lipids in the second trimester for predicting preeclampsia

\begin{tabular}{|c|c|c|c|c|c|c|}
\hline & AUC (95\% Cl) & $\begin{array}{l}\text { Sensitivity } \\
(\%)\end{array}$ & $\begin{array}{l}\text { Specificity } \\
\text { (\%) }\end{array}$ & $\begin{array}{l}\text { Youden } \\
\text { index }\end{array}$ & $\begin{array}{l}\text { Cut-off } \\
\text { point }\end{array}$ & $\begin{array}{l}p \\
\text { value }\end{array}$ \\
\hline FTG & $\begin{array}{l}0.637(0.471- \\
0.803)\end{array}$ & 0.545 & 0.729 & 0.275 & 2.93 & 0.126 \\
\hline $4 \mathrm{~h} \mathrm{TG}$ & $\begin{array}{l}0.680(0.505- \\
0.854)\end{array}$ & 0.727 & 0.65 & 0.378 & 3.92 & 0.045 \\
\hline $\begin{array}{l}\mathrm{HDL}- \\
\mathrm{C}\end{array}$ & $\begin{array}{l}0.419(0.221- \\
0.616)\end{array}$ & 0.182 & 0.897 & 0.078 & 2.42 & 0.221 \\
\hline LDL-C & $\begin{array}{l}0.32(0.154- \\
0.485)\end{array}$ & 1 & 0.049 & 0.049 & 1.8 & 0.154 \\
\hline FFA & $\begin{array}{l}0.511(0.319- \\
0.703)\end{array}$ & 0.182 & 0.99 & 0.172 & 1.46 & 0.903 \\
\hline $\begin{array}{l}\text { sd- } \\
\text { LDL }\end{array}$ & $\begin{array}{l}0.532(0.380- \\
0.685)\end{array}$ & 1 & 0.202 & 0.202 & 29.085 & 0.717 \\
\hline
\end{tabular}

Elevated mid-trimester 4-h postprandial TG concentrations at the time of OLTT measurement as a predictive biomarker for the development of preeclampsia

To determine whether the elevated 4-h postprandial TG concentrations during the second trimester of pregnancy was a predictive biomarker for preeclampsia, the odds ratios (ORs) for preeclampsia in women with different TG concentrations during the second trimester were calculated. First, compared to the low 4-h TG binary group at 12-24 gestational weeks, the crude OR for preeclampsia was $1.471(95 \% \mathrm{Cl}$ $1.049-2.062, p=0.025)$ for the high TG binary group, and the adjusted OR was $5.561(95 \% \mathrm{Cl} 1.189-$ 
26.006, $p=0.029$ ) when considering for age, gravidity, parity, pre-pregnancy BMI, and TC concentrations (Table 5). In addition to the elevated 4-h TG concentrations, we also found that an abnormal OGTT result (OR: $3.869,95 \% \mathrm{Cl}: 1.179-12.697, p=0.026$ ) was a risk factor for preeclampsia. However, no association was found between other factors and preeclampsia.

Table 5

Factors associated with preeclampsia in the logistic regression analysis

\begin{tabular}{|lll|}
\hline & OR $(\% 95 \mathrm{Cl})$ & p value \\
\hline OGTT & & 0.026 \\
\hline Normal & reference & \\
\hline High & $3.869(1.179-12.697)$ & \\
\hline FTG & & 0.238 \\
\hline Low & reference & \\
\hline High & $2.099(0.613-7.187)$ & \\
\hline 4hTG & & 0.029 \\
\hline Low & reference & \\
\hline High & $5.561(1.189-26.006)$ & 0.826 \\
\hline Age (years) & $1.021(0.848-1.230)$ & 0.935 \\
\hline Gravidity & $1.025(0.561-1.874)$ & 0.878 \\
\hline Parity & $0.985(0.814-1.193)$ & 0.681 \\
\hline Prepregnancy BMI (kg/m²) & $1.014(0.948-1.084)$ & 0.946 \\
\hline Total Cholesterol (mmol/L) & $0.971(0.411-2.291)$ & 0.437 \\
\hline HDL-C (mmol/L) & $0.437(0.078-2.442)$ & 0.068 \\
\hline LDL-C (mmol/L) & $0.505(0.243-1.051)$ & \\
\hline $\begin{array}{l}\text { BMI: body mass index; } 95 \% \text { Cl: } 95 \% \text { confidence intervals; FTG: fasting triglyceride; HDL-C: high-density } \\
\text { lipoprotein-cholesterol; LDL-C: low-density lipoprotein-cholesterol; 0GTT: oral glucose tolerance test; } \\
\text { OR: odds ratio; 4h TG: 4-h triglyceride }\end{array}$ & & \\
\hline
\end{tabular}

\section{Discussion}

Early identification of women at a high risk of preeclampsia might enable potential prophylactic treatment to reduce or avoid the onset of symptoms $(9,10)$. Preeclampsia is more common and has a lower detection rate (11). Predictive models for preeclampsia have employed a combination of maternal 
characteristics as well as biochemical and biophysical markers before 14 weeks of gestation to predict the syndrome at $30-60 \%$ sensitivity $(10-14)$.

Our findings are consistent with the results of the few available prospective cohort studies on maternal non-fasting plasma lipid and lipoprotein concentrations in preeclamptic and normotensive pregnancies $(15,16)$. We observed an association between maternal dyslipidemia in the second trimester, particularly 4-h postprandial hypertriglyceridemia, and the subsequent risk of preeclampsia. We evaluated both the FTG and 4-h postprandial TG concentrations at the time of OLTT measurement to show that 4-h postprandial TG concentrations might be an independent predictive biomarker for preeclampsia, which might be more meaningful compared to the FTG concentrations (Table 5). This is in line with the results of a comprehensive study (15) showing the associations between non-fasting plasma TG at 18 weeks of gestation and the risk of preeclampsia. In another prospective cohort study (16), a linear increase in preeclampsia risk was observed with increasing non-fasting plasma TG concentrations before 16 weeks' gestation. However, fasting TG concentrations in the second trimester were not associated with the development of preeclampsia, which was inconsistent with Oya's cohort study findings (OR: 1.1 (1.1-1.2), $p=0.004)(17)$.

Although, the temporality, wherein 4-h postprandial hypertriglyceridemia clearly precedes the onset of preeclampsia, leads us to generate the hypothesis that we may be able to change the natural history of the disease if we intervene early by lowering the postprandial TG concentrations. Maternal lipids offer a potential therapeutic target, and it is worth noting that the current treatments for diabetes in pregnancy, including dietary and lifestyle changes as well as pharmacotherapy, all alter not only maternal glucose but also maternal lipids $(3,18,19)$. Before such an intervention, it would be important to define the normal postprandial triglyceride concentrations in pregnancy and correctly identify women with lipid metabolic disturbances who could benefit most from this therapy.

High postprandial TG concentration is positively correlated with insulin resistance, and insulin resistance exists in preeclampsia patients (Tables 2 and 3). Changes in TG metabolism and clearance are mediated by insulin resistance. Women with elevated lipid concentrations are more likely to have preexisting endothelial dysfunction that worsens as a result of the physiological burden of pregnancy; this condition may be further exacerbated by increased maternal vascular inflammation (20). This was supported with evidence from a murine model, which suggested that, in pregnancy, excessive hyperlipidemia may lead to an accentuated inflammatory response, oxidative stress, and subsequent dysfunction of cerebral arteries, compromising cerebral percussion and contributing to preeclampsia-related neurological complications (21).

The sensitivity of the prediction of preeclampsia using 4-h postprandial TG concentration at the time of OLTT measurement in this study $(72.7 \%)$ was markedly higher than that of previously published prediction of preeclampsia based on clinical markers such as mean arterial pressure, maternal age, and uterine artery pulsatility index in a cohort of women at $11+0$ to $13+6$ gestational weeks (38.5\%) (22) and 
in another nulliparous cohort (37\%) (23). However, the sensitivity at $10 \%$ false positive rate of prediction of preeclampsia is low (18.2\%).

The major strength of our study was its prospective design with complete follow-up of 438 women with OLTT measurement. This study design more accurately reflects the predictive power of 4-h postprandial TG compared to a case-control design. Our high participation (86.6\%) and follow-up (99.5\%) rates minimized the likelihood of selection bias, which may affect the reported results. Second, the fasting and 4-h postprandial blood lipid concentrations obtained after a unified fat meal in the second trimester suggested that the disordered plasma lipid concentrations precede preeclampsia. The unified standard fat meal and blood sampling collection time can avoid lipid data deviation. Third, the exclusion of pregnant women with high risk factors for metabolic syndrome made the results more convincing. Fourth, compared to the fetus in the first trimester, the fetus in the second trimester grows rapidly and needs more energy. The mother gradually switches to a catabolic condition resulting in an increased breakdown of lipid. Therefore, detecting postprandial lipid concentration changes (the ability of lipid metabolism) in the second trimester is more sensitive. Finally, we used the logistic regression to adjust for a number of confounders to obtain a conclusion.

Several important limitations must be considered when interpreting the results of our study. First, our study included a limited number of cases, which is difficult to overcome given the low incidence of these disorders. Our relatively small number of patients with preeclampsia hindered us from making inferences from some of our analyses. Further studies involving multiple centers and a larger number of patients are necessary in order to validate our findings. In addition, although we compared many potential confounders, we cannot exclude the possibility of the other confounding factors from unmeasured covariates.

\section{Conclusions}

In conclusion, the results of our study served to highlight the importance of 4-h postprandial TG at the time of OLTT measurement in the second trimester as a possible predictive marker of preeclampsia. Moreover, dyslipidemia, particularly 4-h postprandial hypertriglyceridemia, may be of etiologic and pathophysiologic importance in women who developed preeclampsia. Our data may contribute to the development and evaluation of behavioral and medical interventions aimed at reducing the occurrence of these disorders. On the other hand, preeclampsia appears to be a multi-etiological syndrome with heterogeneous biologic pathways.

\section{Declarations}

\section{Ethics approval and consent to participate}

The study protocol was approved by the ethics committee of Shanghai General Hospital, Shanghai Jiao Tong University (approval No.: JRS [2018] No.26; approval date: June 20,2018). All patients consented to 
participate this trial.

\section{Consent for publication}

All authors read and consented the final manuscript for publication.

\section{Competing interests}

There are no conflicts of interests to disclose.

\section{Funding}

The present study was supported by the Research Physician Program of Shanghai JiaoTong University (No. 20172020).

\section{Author's contributions}

Qin Liu, Wen Cai and Jiarong Zhang conceived the study question, collected the data and performed the statistical analyses. Shuangdi Li and Liu Yang drafted the first version of the manuscript and it was revised and approved by Qin Liu, Wen Cai, Liu Yang, ShuangDi Li and Jiarong Zhang.

\section{Acknowledgements}

None to declare.

\section{Availability of data and material}

Data and material can be achieved by email to the corresponding author (E-mail address: maggie1974@126.com)

\section{Disclosure statement}

The authors report no conflict of interst.

\section{Clinical trial registration}

1. Data of registration: $2018 / 10 / 15$

2. Date of initial participant enrollment: $2019 / 05 / 01$

3. Clinical trial identification number: chiCTR1800018884

4. URL of the registration site: http://www.chictr.org.cn/showproj.aspx?proj=25526

5. Data sharing information: The data including individual participant data, detailed study protocols, statistical analysis plans will be shared upon request to the corresponding author. 


\section{Sources of support}

The present study was supported by the Research Physician Program of Shanghai JiaoTong University (No. 20172020).

\section{References}

1. Sarosh R, Elizabeth L, Joey PG, S. Ananth K. Preeclampsia Pathophysiology, Challenges, and Perspectives. Circ Res. 2019;124:1094-1112.

2. Brown MC, Best KE, Pearce MS, et al. Cardiovascular disease risk in women with pre-eclampsia: systematic review and meta-analysis. Eur J Epidemiol. 2013;28(1):1-19.

3. Helen LB, Marloes DN, H David M, Leonie KC. Maternal lipids in pre-eclampsia: innocent bystander or culprit? Hypertens Pregnancy. 2014;33(4):508-23.

4. Gallos ID, Sivakumar K, Kilby MD, Coomarasamy A, Thangaratinam S, Vatish M. Pre-eclampsia is associated with, and preceded by, hypertriglyceridaemia: a meta-analysis. BJOG 2013;120:1321-32.

5. Nasioudis D, Doulaveris G, Kanninen TT. Dyslipidemia in pregnancy and maternal-fetal outcome. Minerva Ginecol 2019;71:155-62.

6. Nordestgaard BG, Langsted A, Freiberg JJ. Nonfasting hyperlipidemia and cardiovascular disease. Curr Drug Targets. 2009;10:328-35.

7. Yulong L, Jianxun H, Xiaoli Z, Song Z, Xuebing W, Hui Y. Non-fasting lipids detection and their significance in pregnant women. Lipids Health Dis. 2019;18(1):96-102.

8. A Schmid, S Leszczak, I Ober, A Schäffler, T Karrasch. Serum progranulin concentrations are not responsive during oral lipid tolerance test and oral glucose tolerance test. Horm Metab Res. 2015;47(8):571-6.

9. Stéphanie R, Pia V, Kypros N, Yves G, Merja V, Abdelouahab B, Alaa E, Emmanuel B. Early administration of low-dose aspirin for the prevention of preterm and term preeclampsia: $A$ systematic review and meta-analysis. Fetal Diagn. Ther. 2012, 31(3):141-6.

10. Ranjit A, Argyro S, Leona P, David W, Kypros HN. Competing risks model in early screening for preeclampsia by biophysical and biochemical markers. Fetal Diagn Ther. 2013, 33(1):8-15.

11. Leona CYP, Nikos AK, Nerea M, Ranjit A, Kypros HN. First-trimester prediction of hypertensive disorders in pregnancy. Hypertension 2009, 53:812-8.

12. Ranjit A, Argyro S, Rita S, Mona Z, Kypros HN. Prediction of early, intermediate and late pre-eclampsia from maternal factors, biophysical and biochemical markers at 11-13 weeks. Prenat Diagn. 2011, $31,66-74$.

13. Scazzocchio E, Figueras F, Crispi F, Meler E, Masoller N, Mula R, et al. Performance of a first-trimester screening of preeclampsia in a routine care low-risk setting. Am J Obstet Gynecol. 2013;208(3):203. e1-. e10. 
14. Marie A, Line HT, Ragnhild BS, Kjell AS, Rigmor A, Ann-Charlotte I, Tone FB. First trimester urine and serum metabolomics for prediction of preeclampsia: a prospective screening study. Int J Mol Sci. 2015;16(9):21520-38.

15. Clausen T, Djurovic S, Henriksen T. Dyslipidemia in early second trimester is mainly a feature of women with early onset pre-eclampsia. BJOG 2001;108:1081-7.

16. Enquobahrie DA, Williams MA, Butler CL, Frederick IO, Miller RS, Luthy DA. Maternal plasma lipid concentrations in early pregnancy and risk of preeclampsia. Am J Hypertens 2004;17:574-81.

17. Oya D, Ahmet ST, Nihal D, Hamdullah S, Sadiye E. Serum lipids level assessed in early pregnancy and risk of pre-eclampsia. J Obstet Gynaecol Res. 2011; 37(10): 1427-32.

18. Barrett HL, Dekker Nitert M, Mclntyre HD, Callaway LK. Normalizing metabolism in diabetic pregnancy: is it time to target lipids? Diabetes Care 2014;37:1484-93.

19. Alessandra G, Alessandra B, Veronica R, Laura V, Graziano DC. Triglyceride metabolism in pregnancy. Adv Clin Chem. 2011;55:133-53.

20. Cassandra NS, Caitlin JS, Audrey FS, Jennifer GR, Kelli KR. Maternal hyperlipidemia and the risk of preeclampsia: a meta-analysis. Am J Epidemiol, 2014;180(4):346-58.

21. Schreurs MP, Cipolla MJ. Pregnancy enhances the effects of hypercholesterolemia on posterior cerebral arteries. Reprod Sci. 2013;20:391-9.

22. Ragnhild BS, Gunhild GH, Harm-Gerd KB, Pål RR, Kjell ÅS. A prospective study of screening for hypertensive disorders of pregnancy at 11-13 weeks in a Scandinavian population. Acta Obstet Gynecol Scand. 2014, 93(12), 1238-47.

23. Robyn AN, Lesley MEM, Gustaaf AD, Lucilla P, Eliza HYC, Alistair WS, Michael AB, Rennae ST, James JW, Philip NB, Louise CK. Clinical risk prediction for preeclampsia in nulliparous women:

Development of model in international prospective cohort. BMJ. 2011;342:d1875-85.

\section{Figures}



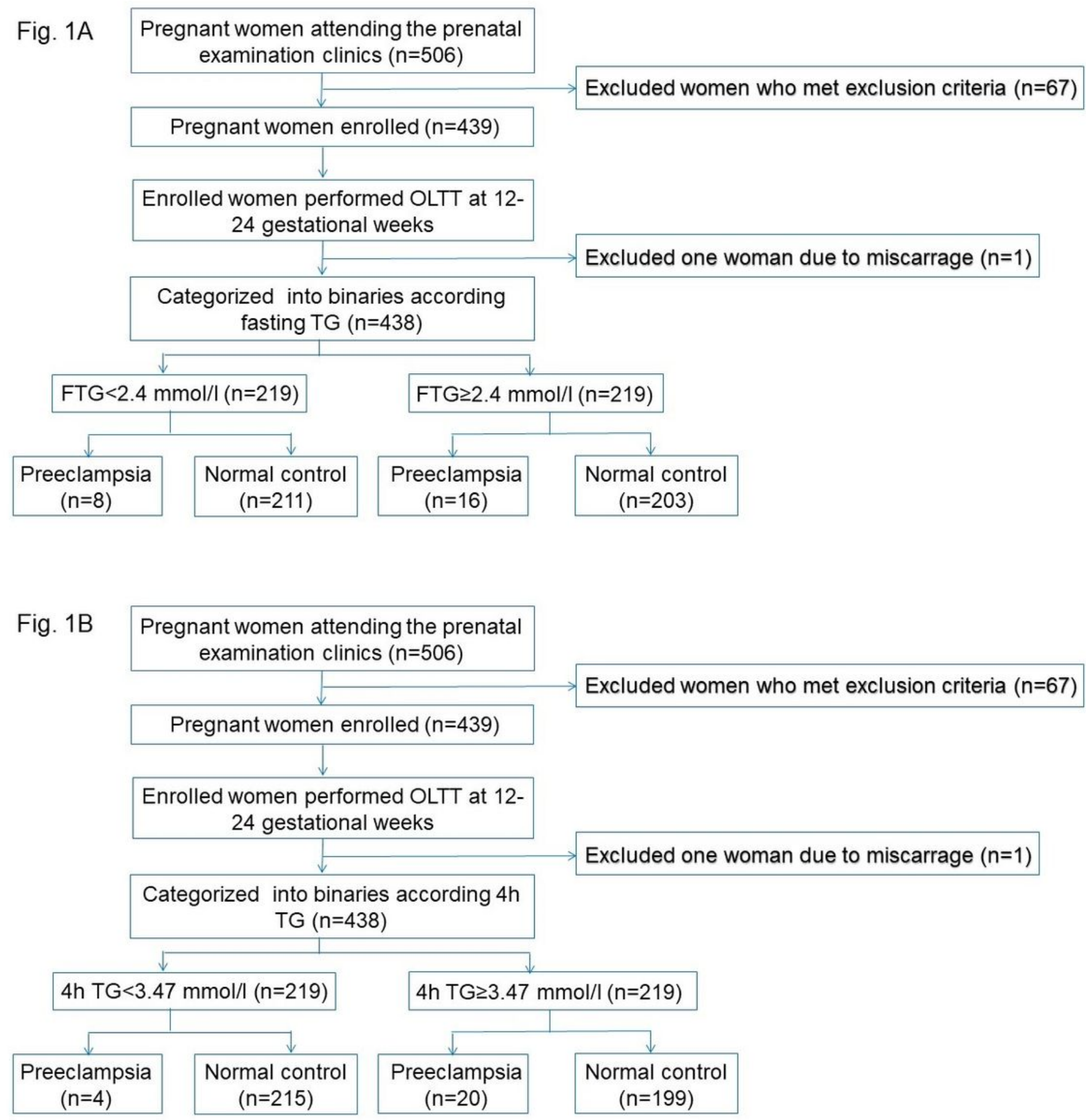

\section{Figure 1}

Subject screening and distribution. A: Subjects distributed by fasting triglyceride. B: Subjects distributed by postprandial triglyceride. 